\title{
Generalized Role for the Cerebellum in Encoding Internal Models: Evidence from Semantic Processing
}

\author{
Torgeir Moberget, ${ }^{1}$ Eva Hilland Gullesen, ${ }^{1}$ Stein Andersson, ${ }^{1,2}$ Richard B. Ivry, ${ }^{3}$ and Tor Endestad ${ }^{1,2}$ \\ ${ }^{1}$ Department of Psychology, University of Oslo, 0316 Oslo, Norway, ${ }^{2}$ Department of Psychosomatic Medicine, Oslo University Hospital, 0317 Oslo, Norway, \\ and ${ }^{3}$ Psychology Department, University of California, Berkeley, Berkeley, California 94720
}

\begin{abstract}
The striking homogeneity of cerebellar microanatomy is strongly suggestive of a corresponding uniformity of function. Consequently, theoretical models of the cerebellum's role in motor control should offer important clues regarding cerebellar contributions to cognition. One such influential theory holds that the cerebellum encodes internal models, neural representations of the context-specific dynamic properties of an object, to facilitate predictive control when manipulating the object. The present study examined whether this theoretical construct can shed light on the contribution of the cerebellum to language processing. We reasoned that the cerebellum might perform a similar coordinative function when the context provided by the initial part of a sentence can be highly predictive of the end of the sentence. Using functional MRI in humans we tested two predictions derived from this hypothesis, building on previous neuroimaging studies of internal models in motor control. First, focal cerebellar activation-reflecting the operation of acquired internal models-should be enhanced when the linguistic context leads terminal words to be predictable. Second, more widespread activation should be observed when such predictions are violated, reflecting the processing of error signals that can be used to update internal models. Both predictions were confirmed, with predictability and prediction violations associated with increased blood oxygenation level-dependent signal in the posterior cerebellum (Crus I/II). Our results provide further evidence for cerebellar involvement in predictive language processing and suggest that the notion of cerebellar internal models may be extended to the language domain.
\end{abstract}

Key words: cerebellum; cognition; fMRI; internal models; language

\section{Introduction}

While there is an emerging consensus on cerebellar involvement in a wide range of cognitive tasks, including language processing (Strick et al., 2009, but see Glickstein et al., 2011), a computational account of how this subcortical structure contributes to cognition remains elusive. Importantly, the striking microanatomical homogeneity of the cerebellum suggests a corresponding unity of function across motor and non-motor domains (Ramnani, 2006), and has inspired the idea that theories developed for motor control should be informative for understanding cerebellar contributions to cognition. One influential theory posits that the cerebellum encodes internal models, neural representations of the essential dynamic properties of an object (e.g., body part or tool) that can be used to predict and control actions involving that object within a particular context (Wolpert et al., 1995; Ito, 2006), or contribute to cognition by similarly encoding the

Received May 27, 2013; revised Jan. 2, 2014; accepted Jan. 8, 2014.

Author contributions: T.M. and T.E. designed research; T.M. and E.H.G. performed research; T.M. and E.H.G. analyzed data; T.M., E.H.G., S.A., R.B.I., and T.E. wrote the paper.

This work is supported by the National Institutes of Health Grant HD060306 (R.B.I) and the US-Norway Fulbright Grant Program (T.M.). We thank Markus Handal Sneve and Haakon Engen for valuable feedback on early drafts of this paper.

The authors declare no competing financial interests.

Correspondence should be addressed to Torgeir Moberget, Department of Psychology, University of 0slo, 0317 0slo, Norway. E-mail: torgeir.moberget@gmail.com.

DOI:10.1523/JNEUROSCI.2264-13.2014

Copyright $\odot 2014$ the authors $\quad 0270-6474 / 14 / 342871-08 \$ 15.00 / 0$ context-specific dynamics of more abstract representations (Ramnani, 2006; Ito, 2008).

Internal models are acquired and formed by supervised, or error-based learning (Doya, 1999): the model is continuously modified if its output, the predicted state of the system, does not match the actual or observed state. Functional MRI (fMRI) studies of motor control have provided evidence of prediction error signals in the human cerebellum (Imamizu et al., 2000; but see Diedrichsen et al., 2005; Schlerf et al., 2012). Moreover, when controlling for error magnitude, changes in more focal cerebellar activation were consistent with the development of an acquired internal model (Imamizu et al., 2000). These findings suggest that activation patterns within the cerebellum reflect the generation of predictions and processing of prediction error signals, two key characteristics of internal models.

Neuroimaging studies of linguistic processing have consistently reported activation within the cerebellum (for review, see Murdoch, 2010). The functional correlates of these activation patterns, however, remain unclear, especially as motor-based accounts such as covert rehearsal have failed to hold up to experimental investigation (Chein and Fiez, 2001; Ravizza et al., 2006). Interestingly, the neuroscience of language has in recent years increasingly focused on predictive mechanisms (Poeppel et al., 2012), either treating language within the broader theoretical context of predictive coding (Gagnepain et al., 2012) or explicitly using the concept of internal models (Pickering and Garrod, 2013). 


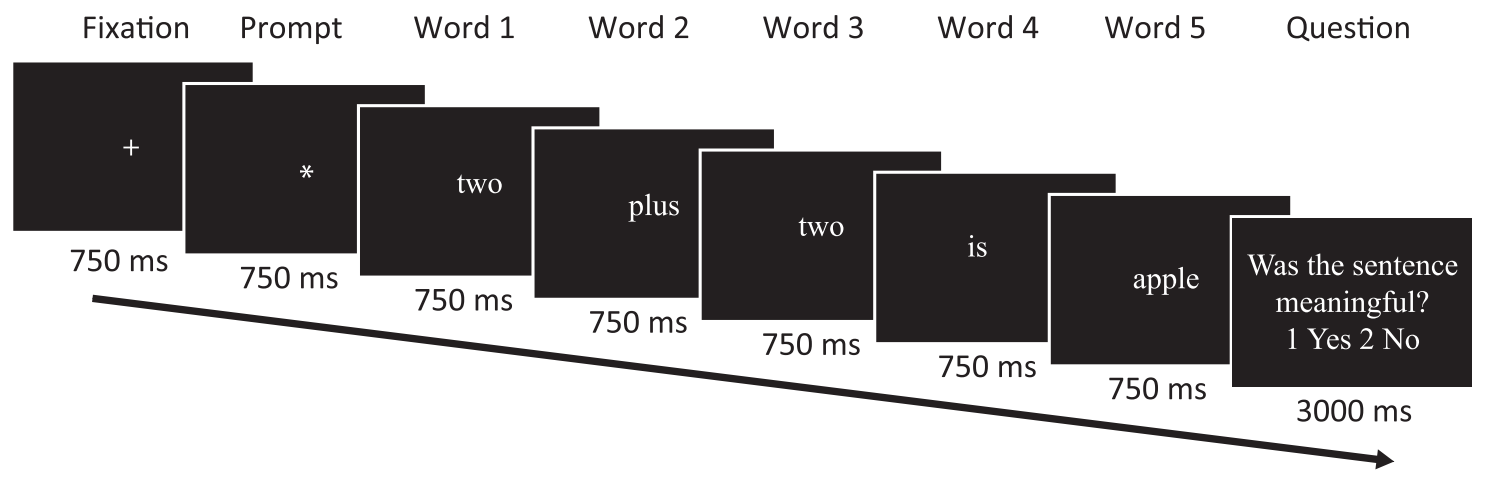

Figure 1. Schematic of the trial structure for a trial in the Incongruent condition.

Thus, our aim in the current study was to test whether the notion of internal models can help shed light on the cerebellar role in language processing. Specifically, we reasoned that cerebellar internal models might aid sentence comprehension, by using the context of a partially presented sentence to predict the next word. A recent transcranial magnetic stimulation (TMS) study has provided support for this generalization of the internal model hypothesis to language (Lesage et al., 2012). Here, we use fMRI to obtain converging evidence and test additional predictions of this hypothesis.

Building on neuroimaging studies examining the role of the cerebellum in internal models for motor control, we tested two key predictions: (1) focal cerebellar activation, reflecting the engagement of a learned internal model, should be observed when the sentence context makes the final word predictable and (2) more widespread activation should be observed when this prediction is violated, reflecting the processing of error signals that can be used to update the internal model or create a novel internal model.

Based on recent meta-analyses, we expected these activations in the posterior cerebellum, predominantly in the right cerebellar hemisphere (Stoodley and Schmahmann, 2009; E et al., 2014).

\section{Materials and Methods}

Participants. All participants were self-reported as right-handed, had normal or corrected-to-normal vision, no known neurological deficits, and were fluent in Norwegian. Of the 39 participants recruited for the study, two were excluded due to abnormalities discovered on MRI, and five others due to excessive head movement in the scanner, leaving a final sample of 32 (21 female, mean age $26.2(S D=9.08)$ ). All participants provided oral and written informed consent. The study was approved by the Regional Ethics Committee and was conducted in accordance with ethical standards specified in the 1964 Declaration of Helsinki.

Experimental procedure. An illustration of the task structure is given in Figure 1. Briefly, on each trial, the participant viewed a fixation cross, followed by a visual prompt (asterisk) and a sequence of five centrally presented words (in lower case). Each of these stimuli was presented for $750 \mathrm{~ms}$, and there was no pause between successive stimuli $(0 \mathrm{~ms}$ interstimulus interval). We used a fixed rate of stimulus presentation to minimize the disruptive effects of serial reading, while placing minimal demands on working memory.

The crucial experimental variable, the predictability of the terminal, target word, was manipulated by varying the context established by the initial four words. In the Congruent condition, sentences were constructed so that the target word was highly predictable (e.g., "two plus two is four."). In the Incongruent condition, the sentences were also designed such that the target word was highly predictable, but the prediction was violated by presenting a terminal word that was inappropriate given the context (e.g., "[the water] had frozen to cars"). In the Scrambled condition, the initial four words did not establish a context for a grammatical sentence (e.g., "fast in clock plane"), and thus the target word was not predictable (e.g., "through"). We also included a Letter String condition to control for the visual and motor aspects of the task, replacing the words with meaningless letter strings of identical consonants (e.g., "rrr gggg nnnn pp kkkk").

Immediately after the presentation of the target word (or consonant string), the question, "Was the sentence meaningful?" was presented on the screen, indicating that the participant should judge whether or not the sequence constituted a meaningful sentence (Congruent condition vs Incongruent, Scrambled, and Letter String conditions). This question was displayed for $3000 \mathrm{~ms}$ and the participant was required to respond within this time window by pressing one of two buttons with his/her right hand, using the index finger ("yes") or thumb ("no"). Participants were instructed to wait for the question before answering, and were told that there was no need to respond quickly. The onset of the next trial followed directly after the offset of the question.

The entire experiment consisted of 30 trials per condition, plus 15 null trials in which an asterisk replaced the words/letters for the full trial duration. The order of the 135 trials was randomized. Stimuli were presented using E-Prime 2.0 software (Psychology Software Tools) and MR-compatible goggles with two LCD-displays (VisualSystems; NordicNeuroLab), while responses were collected using an MR-compatible response grip with two response buttons (ResponseGrip; NordicNeuroLab). The total duration of the single functional scanning run was $\sim 19 \mathrm{~min}$.

The sentence stimuli used in the present experiment were constructed with the aim of maximizing the predictability of the final word in the congruent and incongruent conditions, with the additional constraint that the presented target word in the incongruent condition constitute a violation of these predictions. We confirmed this by presenting 100 participants with the context phrase (four initial words) for the 30 congruent and 30 incongruent sentences and asking them to generate a terminal, target word. Cloze probability, the ratio of participants who used the actual target word to complete the sentences, was 0.85 (SD: 0.19) for congruent sentences and 0 for incongruent sentences. Word frequency, defined as the number of occurrences per million words, was extracted from a large database of Norwegian words (The Text Laboratory, ILN, University of Oslo; http://www.tekstlab.uio.no/frekvensordlister/).

A repeated-measures ANOVA was conducted to compare the conditions in terms of word frequency given that this variable is known to influence the blood oxygenation level-dependent (BOLD) response (Chee et al., 2002; Carreiras et al., 2006; Grande et al., 2011). When all five words were included, there was no significant difference between conditions in word frequency $\left(F_{(2,447)}=1.309, p=0.271\right)$. However, if the analysis is restricted to the target words, the effect of condition was reliable $\left(F_{(2,87)}=3.944, p<0.05\right)$, with the difference occurring because a number of high-frequency function words (e.g., "and" and "in") appeared in the target position for some of the "sentences" in the scrambled condition. Given the frequency differences, a post hoc procedure was applied to the stimulus sets to equate the conditions for target word frequency. We excluded six to eight sentences for each condition, eliminating those sentences in which the target word was at either extreme in terms of frequency (high or low frequency). This approach effectively equalized mean word frequency across conditions $\left(F_{(2,67)}=0.241\right.$, 
$p=0.787$; Means: Congruent: 16.56 [13.54]; Incongruent: 15.04 [15.83]; Scrambled: 13.27 [18.46]), with the penalty of slightly reducing power by reducing the number of trials per condition. All fMRI analyses were performed both with the full set of sentences and with the pruned sentence sets in which word frequency was better controlled.

Scan acquisition. Scanning was conducted on a 3 T, Phillips Achieva whole-body scanner, with an 8 channel Philips SENSE head coil (Philips Medical Systems). Functional images were obtained with a single-shot $\mathrm{T} 2{ }^{\star}$ weighted echo planar imaging sequence (repetition time (TR): 2000 ms; slice echo time (TE): $30 \mathrm{~ms}$; field of view (FOV): $240 \times 240 \times 108$; imaging matrix: $80 \times 80$; flip angle $80^{\circ} 36$ axial slices, interleaved at $3 \mathrm{~mm}$ thickness, no gap, voxel size $3 \times 3 \times 3 \mathrm{~mm}$ ). The scanning session consisted of 563 volumes, synchronized to the onset of the experiment. To obtain complete coverage of the cerebellum, the slice orientation was adjusted to be $\sim 45^{\circ}$ relative to the line running from the anterior to posterior commissure. This orientation resulted in parts of the posterior frontal lobe and superior parietal lobe falling outside the FOV. A T1 weighted anatomical image with a voxel size of $1 \times 1 \times 1 \mathrm{~mm}$ was recorded for registration of the functional images ( 180 sagittal slices; TR: $8.5 \mathrm{~ms}$; TE: $2.3 \mathrm{~ms}$; FOV: $256 \times 256 \times 180$; flip angle: $\left.7^{\circ}\right)$.

Imaging analysis. Functional images were converted to $4 \mathrm{D}$ NIfTI files (http://lcni.uoregon.edu/ jolinda/MRIConvert/) and analyzed using SPM8 (http://www.fil.ion.ucl.ac.uk/spm/software/spm8). Images were corrected for slice timing, realigned to correct for residual head movement, and coregistered to the anatomical image. Following these preprocessing steps, the analysis stream was split into a cerebellum-specific analysis to address the main hypotheses of the study, and a whole-brain analysis to examine cerebral activation patterns.

For the cerebellum-specific analysis, unsmoothed images were first analyzed in native space using a general linear model (GLM). Eventrelated regressors, modeled as delta functions time locked to the onset of the target word, were created for the four trial types (congruent, incongruent, scrambled, letter strings). These functions were convolved with the canonical hemodynamic response function. Low-frequency drifts were removed using a high-pass filter (cutoff $128 \mathrm{~s}$ ) and six head motion parameters from the realignment step were included as additional regressors. Serial correlations in fMRI time series were accounted for by the autoregressive AR(1) model.

The anatomical images were normalized to a high-resolution cerebellar template Spatially Unbiased Infratentorial Template (SUIT; Diedrichsen, 2006) allowing us to bring the functional contrast images (the weighted sums of single $\beta$-images) into a common template space, resliced to $2 \times 2 \times 2 \mathrm{~mm}$ voxels. The normalized contrast images were smoothed with a 3D Gaussian kernel (4 mm full-width at half-maximum; FWHM). Statistical analyses were performed using randomeffects analyses on these images.

For the whole-brain analysis, we normalized the anatomical images to the MNI template using the unified segmentation and normalization algorithm implemented in SPM8 (Ashburner and Friston, 2005). The resulting transformation parameters were then applied to the functional images. Images were smoothed with a Gaussian kernel of $8 \mathrm{~mm}$ FWHM and analyzed using the same GLM as was used in the cerebellum-specific analysis.

We recognize that the presentation of single words can be sufficient to generate predictions of, or prime, related words (Meyer and Schvaneveldt, 1971); indeed, presentations of pairs of unrelated words elicits an electrophysiological response (N400) associated with semantic prediction errors (Ortu et al., 2013). Consequently, the Scrambled condition is likely to involve predictions and prediction errors to some degree. Note, however, that the crucial comparisons in the present study involve the final target word and that extensive research on the N400 response (for review, see Kutas and Federmeier, 2011) has shown that predictability in higher level language structure (such as sentences or discourse) can amplify and even override lower level priming effects (e.g., word-pair associations or repetition priming). Based on these findings, we assume that predictions generated in response to contextually isolated words in the Scrambled condition are considerably weaker (i.e., less precise) than the strong predictions generated after the presentation of the initial four words in the Congruent and Incongruent conditions (see also the behav- ioral validation of prediction strength, i.e., cloze probability, below). Thus, our main focus for assessing predictions and violations of predictions will be on the comparison of the Congruent and Incongruent conditions.

Even though response speed was de-emphasized in the current experiment, we expected we would observe reaction time (RT) differences between the experimental conditions (Debruille and Renoult, 2009). RT differences between conditions can influence the resulting activation patterns in at least two ways (Grinband et al., 2008). First, neural activity related to the actual response (motor preparation, sensory feedback) can be shifted in time between short and long RTs, potentially affecting the hemodynamic response to the preceding stimuli in differential ways. Second, RT differences might reflect differences in time spent on the task, leading to a larger summation of the BOLD response for conditions associated with long RTs (Grinband et al., 2008). We addressed this issue using a two-step approach. First, we included the single trial RTs as additional regressors in the first-level analysis, using these values to modulate each of the four condition regressors. That is, each condition was modeled by a constant regressor representing the task condition, and an orthogonalized regressor that accounted for effects of trial-by-trial variability in RT. Second, since mean RT would still be reflected in the constant condition regressor, we also calculated, on an individual basis, the mean RT difference between any pair of contrasted conditions. The difference scores were then included as a covariate in the group analyses of the contrast images. Importantly, this approach makes no assumptions regarding the nature or consequences of RT differences (e.g., temporal shift or differential time on task). Rather, the procedure assumes that, if such differences do influence the BOLD response, these effects will be captured in the regressors modeling trial-by-trial variability in RT, as well as in the second-level covariates modeling mean RT-differences between conditions.

A significance level of 5\% (FDR corrected for multiple comparisons) was adopted for all analyses. To this end, we set a voxelwise clusterforming threshold of $p<0.005$ (uncorrected), with statistical significance assessed by evaluating the volume of the active clusters (Chumbley and Friston, 2009). Unthresholded statistical maps were uploaded to the NeuroVault.org database and are available at http://neurovault.org/ collections $/ 25 /$.

\section{Results}

\section{Behavior}

Overall accuracy across conditions was $96 \%$, indicating that participants judged congruent sentences as meaningful, and judged incongruent sentences, scrambled words, and letter strings as meaningless. As expected, RTs differed considerably across conditions, $\left(F_{(3,93)}=17.779, p<0.0001\right)$. RTs were much faster in the Congruent condition (mean: 704.3; SD: 260.3) compared with the Incongruent condition (mean: 900.9; SD: 309.0). The means for the Scrambled (mean: 783.7; SD: 358.4) and Letter String conditions (mean: 807.3; SD: 227.7) fell between the means for the congruent and incongruent conditions. Note that participants in the scrambled and control condition could anticipate their response well in advance of the target word.

\section{RT effects on the BOLD signal}

Figure $2 a$ depicts regions in which the BOLD response was sensitive to trial-to-trial variability in response time, averaged over the four RT modulator regressors. This analysis revealed significant positive relationships in a number of brain areas, indicating a pattern of increased BOLD response with increased RT. Consistent with previous studies, this effect was most evident in medial frontal cortex (Yarkoni et al., 2009; Grinband et al., 2011), as well as anterior insula, prefrontal and parietal cortices, and several subcortical areas (Yarkoni et al., 2009). The cerebellar-specific analysis confirmed RT-related effects in several cerebellar clusters. No regions showed a negative relationship between the BOLD response and RT. 
a
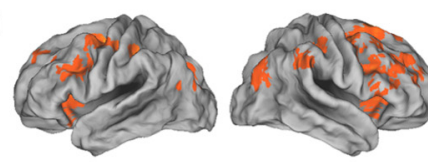

$t=2.75 \quad t=7$
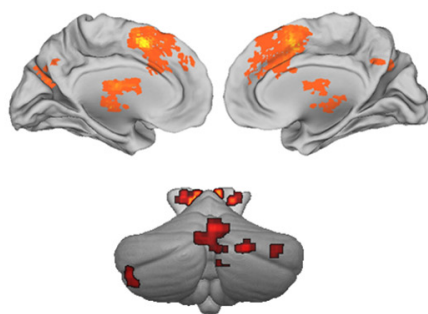

$t=2.75$ b
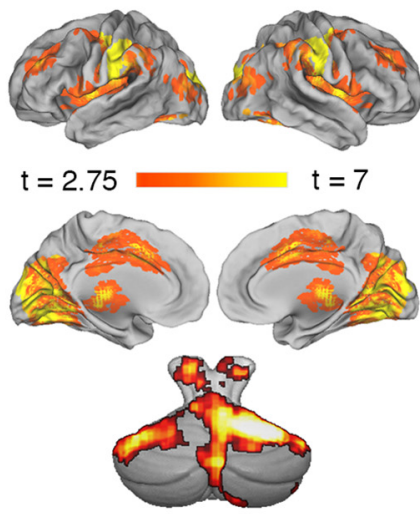

$t=2.75$
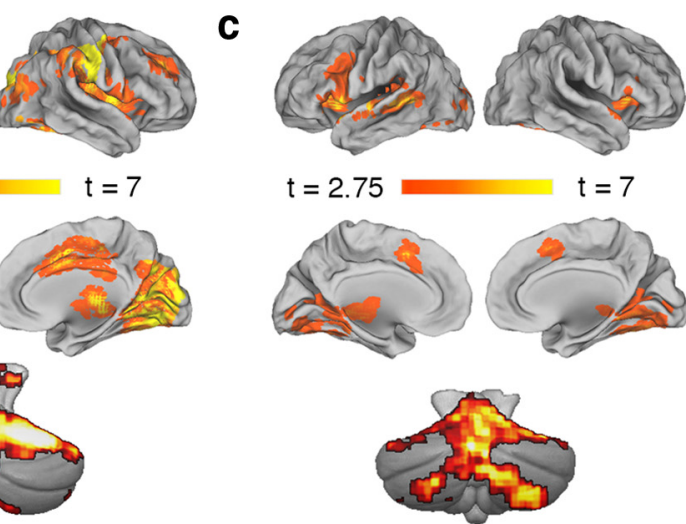

$t=2.75$

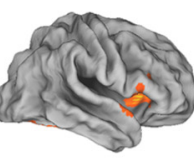

$\mathrm{t}=7$

Sensorimotor

Word Processing

Figure 2. BOLD activations in control analyses. $\boldsymbol{a}$, Regions showing a positive correlation between RT and BOLD response. $\boldsymbol{b}$, Sensorimotor activations, revealed by the contrast of Letter Strings $>$ implicit experimental baseline. The cerebellar template has been oriented to reveal the peak activation in the superior cerebellum (lobule V). $c$, Regions involved in word processing, revealed by the contrast of Scrambled $>$ Letter Strings. Images are thresholded at $p<0.005$ (uncorrected) and show clusters surviving cluster-level FDR correction for multiple comparisons $(q<0.05)$. Cerebral activations are displayed on semi-inflated brain templates using the Caret software package (Van Essen, 2012), while cerebellar activations are displayed on the SUIT template (Diedrichsen, 2006) using MRIcron (Rorden et al., 2007). Complete unthresholded $t$-maps from all reported analyses can be downloaded from http://neurovault.org/collections/25/.

BOLD activations related to visuomotor aspects of the task and processing of single words

Figure 2 further show cerebellar and cerebral activations in (b) the Letter String (perceptual control) condition relative to the implicit experimental baseline and (c) the contrast of Scrambled words versus Letter Strings. As expected, the Letter String condition primarily activated areas involved in visual perception and motor control-including strong activations in cerebellar lobules V and VIII. Areas showing greater activation in the Scrambled condition compared with the Letter String condition were predominantly left-lateralized cerebral areas associated with linguistic processing (Price, 2010), as well as extensive-primarily right-lateralized-cerebellar activations.

\section{Contextual priming effects on the cerebellar BOLD response}

Figure 3 and Table 1 show the results related to the core hypotheses of this study. We first examined the effects of predictability on the cerebellar BOLD signal by contrasting the Congruent and Scrambled conditions (here and elsewhere, regressing out the effect of RT). This analysis revealed a significant cluster in the right posterior cerebellum, Crus I and II (Fig. $3 a$ ), with the BOLD response larger when the context made the target word predictable. While additional clusters were also observed in right lobule IX and bilateral brainstem, these did not remain significant when the analysis was restricted to sentences in which word frequency was equated between conditions.

To examine the effects of violating predictions in sentence comprehension, a contrast was performed between the Incon- b

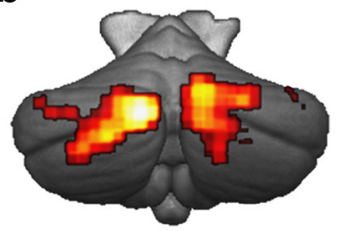

Incongruent > Congruent
C

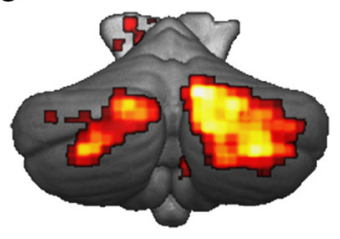

Incongruent $>$ Scrambled

Figure 3. Cerebellar regions sensitive to contextual predictability. $\boldsymbol{a}$, Activations related to contextually predictable words revealed by the contrast of Congruent $>$ Scrambled. $\boldsymbol{b}$, Activations related to violations of predictability, revealed by the contrast of Incongruent $>$ Congruent. c, Activations revealed by the contrast of Incongruent $>$ Scrambled. $\boldsymbol{d}$, Secondary tests for areas sensitive to prediction based on the contrasts of Incongruent $>$ Scrambled (red), Congruent $>$ Scrambled (green), and their overlap (yellow). $\boldsymbol{e}$, Axial slice showing the activation patterns attributed by Imamizu et al. (2000) to error processing (red), an acquired internal model of a new tool (blue), and their overlap (orange). Reprinted with permission.

gruent and Congruent conditions. This analysis revealed greater activation in the Incongruent condition across an extensive region of the posterior cerebellum, bilaterally (Fig. $3 b$ ). We also looked at hemodynamic correlates of prediction violations by comparing the Incongruent and Scrambled conditions. Notably, in both of these conditions the actual target word is unpredictable. However, only in the Incongruent condition does the presentation of the target word constitute a violation of a (strong) prediction, established by the preceding context. Consistent with the contrast of the Incongruent and Congruent conditions, cerebellar activation was stronger in the Incongruent condition compared with the Scrambled condition, and these effects were also bilateral (Fig. 3c). 
Table 1. MNI coordinates of contextually modulated cerebellar and brainstem activation clusters

\begin{tabular}{|c|c|c|c|c|c|c|c|}
\hline \multirow[b]{2}{*}{ Contrast } & \multirow[b]{2}{*}{ Volume $\left(\mathrm{cm}^{3}\right)$} & \multirow[b]{2}{*}{$q$ (corr.) } & \multirow[b]{2}{*}{$Z$} & \multicolumn{3}{|c|}{ Coordinates } & \multirow[b]{2}{*}{ Cerebellar lobules } \\
\hline & & & & $x$ & $y$ & $z$ & \\
\hline \multirow[t]{4}{*}{ Congruent $>$ Scrambled } & 2.57 & 0.000 & 3.75 & 32 & -74 & -37 & Right Crus I/II \\
\hline & 1.06 & 0.010 & 4.27 & 8 & -54 & -45 & Right lobule IX* \\
\hline & 1.06 & 0.010 & 4.08 & -20 & -10 & -3 & Left brainstem (thalamus, red nucleus)* \\
\hline & 0.66 & 0.044 & 4.37 & 6 & -16 & -7 & Right brainstem (thalamus, red nucleus) \\
\hline \multirow[t]{2}{*}{ Incongruent $>$ Congruent } & 7.87 & 0.000 & 5.40 & -10 & -84 & -31 & Left Crus I/II, IV, vermis Crus I \\
\hline & 7.77 & 0.000 & 4.65 & 18 & -74 & -29 & Right Crus I/II, vermis Crus I/II, VI \\
\hline \multirow[t]{4}{*}{ Incongruent $>$ Scrambled } & 14.36 & 0.000 & 5.34 & 22 & -78 & -29 & Right Crus I/II, vermis Crus I/II, VI \\
\hline & 5.72 & 0.000 & 4.49 & -16 & -80 & -29 & Left Crus I/II, vermis Crus I \\
\hline & 1.90 & 0.001 & 5.41 & 6 & -54 & -41 & Right IX, left IX, vermis VIIla/b, IX \\
\hline & 1.13 & 0.009 & 4.50 & -8 & -20 & -7 & Left brainstem (thalamus, red nucleus) \\
\hline
\end{tabular}

Clusters of activation for contrasts of interest. We present the volume of the cluster (thresholded at voxelwise $p<0.005$, uncorrected), FDR-corrected cluster-level $p$ value, the peak voxel $z$ values within the cluster, the MNI coordinates (in SUIT space; Dietrichsen, 2006), and the anatomical extent of the cluster (in lobules; Dietrichsen et al., 2006). Anatomical labels in bold correspond to the MNI coordinates of peak activations. Activations marked with an asterisk failed to reach statistical significance in the control analyses where word frequency was equalized across conditions.

Congruent sentences $>$ Scrambled words
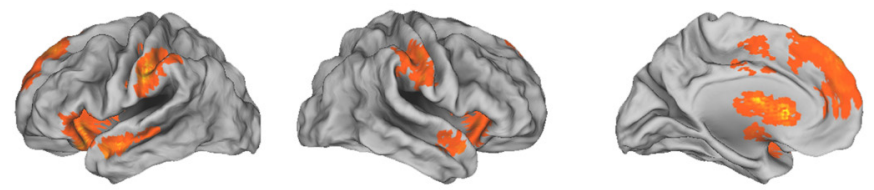

Incongruent sentences $>$ Congruent sentences
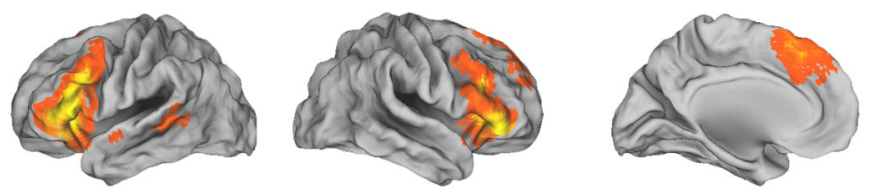

Incongruent sentences $>$ Scrambled words
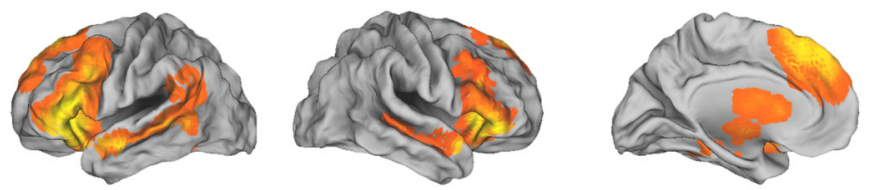

$\mathrm{t}=2.75$

Figure 4. Cerebral regions sensitive to contextual predictability.

In addition to providing an assay on prediction violations, the comparison of the Incongruent and Scrambled conditions provides a second probe on regions correlated with the generation of predictions, given the assumption that the first four words of the Incongruent condition allows for the generation of a strong (but violated) prediction, whereas the Scrambled condition does not. As shown in Figure $3 d$, there was substantial spatial overlap between the Congruent $>$ Scrambled and Incongruent $>$ Scrambled contrasts, with the extent of activation greater in the latter contrast.

\section{Contextual priming effects on cerebral BOLD signal}

Figure 4 and Table 2 present the results from the whole-brain analysis for activations in the cerebral cortex. For the contrast examining prediction generation, activation was greater in the Congruent compared with Scrambled condition in bilateral superior medial cortex, bilateral inferior frontal gyrus, bilateral supramarginal/postcentral cortex, and left middle temporal gyrus, as well as bilateral caudate, thalamus, and brainstem. A broadly
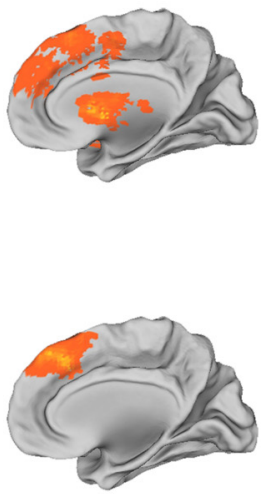

similar pattern was observed in the contrast of the Incongruent versus the Congruent condition, although the effects were stronger and also extended into dorsolateral prefrontal cortex. Contrasting the Incongruent over the Scrambled condition confirmed that violations of semantic predictions produces a broad activation pattern, with the BOLD signal significantly greater in superior medial frontal cortex, bilateral inferior frontal gyri, and lateral prefrontal cortex, as well as in the left posterior middle temporal gyrus, left fusiform gyrus, and left angular gyrus.

\section{Discussion}

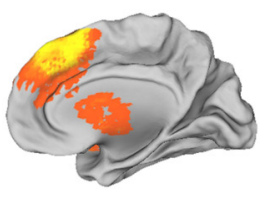

Various lines of evidence have implicated the cerebellum in language processing, with the most compelling evidence coming from an extensive body of neuroimaging studies showing cerebellar activation during a range of linguistic tasks (for review, see Murdoch, 2010). Several functional hypotheses have been proposed, including ideas that highlight languagespecific functions such as the activation of potential phonological codes (Nicolson et al., 2001), covert articulation (Chen and Desmond, 2005), and the coordination of lexical search (Desmond et al., 1998), to more generic ideas, for instance, concerning a role for the cerebellum in supporting attentional shifts (Allen et al., 1997). Here we build on the hypothesis that the cerebellum plays a central role in predictive behavior, learning internal models that can be used to generate context-specific expectancies (Ramnani, 2006), exploring a potential functional parallelism across the domains of motor control and language. Our extension of the internal model hypothesis to language is further grounded in the observation that, despite its generative capacity, language is highly redundant, with communication facilitated through the predictive interactions between speaker and listener (Pickering and Garrod, 2007).

We tested this hypothesis using a contextual semantic priming task. As hypothesized, the BOLD signal in the right posterior cerebellum increased when the target word was predictable. We recognize that, while cerebellar activation was modulated by predictability, the actual predictions could be generated elsewhere in 
Table 2. MNI coordinates of contextually modulated cerebral activation clusters

\begin{tabular}{|c|c|c|c|c|c|c|c|}
\hline \multirow[b]{2}{*}{ Contrast } & \multirow{2}{*}{$\begin{array}{l}\text { Volume } \\
\left(\mathrm{cm}^{3}\right)\end{array}$} & \multirow[b]{2}{*}{$q$ (corr.) } & \multirow[b]{2}{*}{$z$} & \multicolumn{3}{|c|}{ Coordinates } & \multirow[b]{2}{*}{ Anatomical labels } \\
\hline & & & & $x$ & $y$ & $z$ & \\
\hline \multirow[t]{7}{*}{ Congruent $>$ Scrambled } & 62.05 & 0.000 & 5.04 & -9 & 17 & 5 & L/R caudate nucleus, L/R putamen, L hippocampus, brainstem \\
\hline & & & 4.66 & -30 & 20 & -13 & $\begin{array}{l}\text { L lateral orbitofrontal gyrus, L insula, L inferior frontal gyrus, L gyrus rectus, } \\
\text { L middle orbitofrontal gyrus }\end{array}$ \\
\hline & & & 4.56 & -57 & -4 & -19 & L middle temporal gyrus, L superior temporal gyrus \\
\hline & & & 3.92 & 33 & 20 & -7 & R insula \\
\hline & 13.18 & 0.000 & 4.84 & -60 & -22 & 32 & L supramarginal gyrus, L postcentral gyrus, L superior parietal gyrus \\
\hline & 43.15 & 0.000 & 4.56 & -9 & 53 & 38 & L/R superior frontal gyrus, L/R cingulate gyrus, L middle frontal gyrus \\
\hline & 6.94 & 0.000 & 4.30 & 57 & -22 & 44 & R supramarginal gyrus, R postcentral gyrus \\
\hline \multirow[t]{4}{*}{ Incongruent $>$ Congruent } & 29.54 & 0.000 & 5.91 & -10 & -84 & -29 & $\begin{array}{l}\text { L inferior frontal gyrus, L middle frontal gyrus, L lateral orbitofrontal gyrus, } \\
\text { L insula, L middle orbitofrontal gyrus, L superior temporal gyrus }\end{array}$ \\
\hline & 16.82 & 0.000 & 5.45 & 18 & -74 & -29 & $\begin{array}{l}\text { R lateral orbitofrontal gyrus, R inferior frontal gyrus, R middle frontal gyrus, } \\
\text { R middle orbitofrontal gyrus, R insula, R superior temporal gyrus }\end{array}$ \\
\hline & 14.90 & 0.000 & 4.77 & 9 & 29 & 41 & L/R superior frontal gyrus, $R$ middle frontal gyrus \\
\hline & 2.11 & 0.005 & 4.31 & -54 & -40 & 8 & L superior temporal gyrus, L middle temporal gyrus \\
\hline \multirow[t]{8}{*}{ Incongruent $>$ Scrambled } & 134.60 & 0.000 & 6.18 & -51 & 32 & 8 & $\begin{array}{l}\text { L inferior frontal gyrus, L lateral orbitofrontal gyrus, L middle orbitofrontal gyrus, } \\
\text { L insula, L middle frontal gyrus }\end{array}$ \\
\hline & & & 4.81 & -12 & 17 & 5 & L/R caudate \\
\hline & & & 5.81 & -48 & -4 & -19 & L superior temporal gyrus, L middle temporal gyrus \\
\hline & & & 3.91 & -33 & -37 & -19 & L fusiform gyrus, L inferior temporal gyrus \\
\hline & & & 4.29 & -39 & -58 & 23 & L angular gyrus, $\mathrm{L}$ supramarginal gyrus \\
\hline & & & 5.51 & 39 & 32 & -7 & $\begin{array}{l}\text { R inferior frontal gyrus, R lateral orbitofrontal gyrus, R insula, R middle frontal gyrus, } \\
\text { R middle orbitofrontal gyrus }\end{array}$ \\
\hline & & & 4.22 & 48 & -31 & -7 & R middle temporal gyrus, $R$ superior temporal gyrus \\
\hline & 47.09 & 0.000 & 5.81 & 0 & 44 & 50 & L/R superior frontal gyrus, $L / R$ middle frontal gyrus, $L / R$ cingulate gyrus \\
\hline
\end{tabular}

Clusters of activation for contrasts of interest. We present the volume of the cluster (thresholded at voxelwise $p<0.005$, uncorrected), FDR-corrected cluster-level $p$ value, voxel $z$ values, and MNI coordinates of peak activations within each cluster and the anatomical extent of the cluster (from the LONI probabilistic atlas; Shattuck et al., 2008). Anatomical labels in bold correspond to the MNI coordinates of peak activations. L, left; $R$, right.

the brain; indeed, the whole-brain analysis revealed that a predictable linguistic context engaged a broad network of cortical and subcortical areas (Fig. 4, Table 3). Importantly, Lesage et al. (2012) recently provided compelling TMS evidence for a role of the cerebellum in the generation of linguistic predictions. Participants listened to spoken sentences and were required to look, as quickly as possible, at one of four pictures that corresponded to the last word. The sentences either provided a context that strongly predicted the final word or created a context in which all of the pictures were equally plausible. Crucially, repetitive TMS applied over the right cerebellar hemisphere selectively slowed saccade RTs in the predictive condition. A domain-general role of the cerebellum in predictive functions is further supported by studies demonstrating that patients with cerebellar lesions show impairments on tests of predictive motor control (Bastian, 2006) and abnormal effects of predictability on electrophysiological measures of basic auditory processing (Knolle et al., 2012, 2013).

Our second main finding was the increased cerebellar activation when contextual expectancies were violated. Error-based learning has been a central tenet of cerebellar computational theories (Marr, 1969; Albus and Branch, 1971; Doya, 1999; Ito, 2006). Consistent with this idea, cerebellar patients show deficits on sensorimotor adaptation tasks (Tseng et al., 2007), where errors experienced during one trial lead to corrective adjustments in the motor output on the next trial (Wolpert et al., 2011). Furthermore, error-related increases in BOLD have been observed in the cerebellum in studies of sensorimotor control (Schlerf et al., 2012) and learning (Imamizu, 2000; Imamizu and Kawato, 2012), as well as during the perception of visual sequences (Bubic et al., 2009). The present findings extend this line of research by providing evidence for cerebellar error-related activity during language processing. While not the main focus of the current study, the cerebral activation patterns were in general agreement with previous studies investigating the processing of semantic violations (Lau et al., 2008).
The cerebellar results in our language task showed intriguing similarities with those previously reported in an fMRI study of sensorimotor learning (Fig. $3 e$; Imamizu et al., 2000). In the motor study, a spatially widespread activation pattern was observed at the start of training, correlated with the magnitude of performance errors. This activation decreased, both in intensity and extent, with learning. However, even after prolonged learningand a stabilization of error magnitude-a focal area of activation remained, which we attributed to the recruitment of an acquired internal model (Fig. 3b). These results cannot be directly compared with the present findings, given that language regularities are highly overlearned, unlike the novel tool used in the Imamizu study. Nonetheless, together, the results suggest similar cerebellar activation dynamics across task domains.

The current findings are in general agreement with previous studies of cerebellar involvement in language processing. As noted above, Lesage et al. (2012) were able to disrupt anticipatory responses due to sentential priming by applying rTMS over the right cerebellar hemisphere. Using magnetic encephalography, Kujala et al. (2007) found increased coherence between the cerebellum and the left temporal pole when participants read meaningful sentences compared with reading the same words in scrambled order. Similarly, an fMRI study reported increased activation in the right posterior cerebellum when subjects read sentences and narratives relative to the same words presented in random sequences (Xu et al., 2005). In all of these studies, the critical variable was the predictability of the presented wordswith increased cerebellar involvement for predictable relative to unpredictable conditions.

More generally, neuroimaging (Stoodley and Schmahmann, 2009; Buckner et al., 2011) and neuroanatomical (Strick et al., 2009) studies have linked the cerebellar areas activated in the current study to higher cognitive function. For example, Balsters et al. (2013) observed activation changes in these areas when people were required to use abstract higher order rules. Rule- 
based reasoning likely entails some degree of linguistic encoding. Conversely, language, by its nature, entails complex, hierarchically nested representations, similar to the kinds of representations required for understanding abstract, higher order rules. An interesting question for future research will be to design tasks that seek to compare the internal model and rule representation hypotheses. Perhaps cerebellar activation observed when people are asked to reason with complex rules reflects the operation of an anticipatory thought process required to simulate possible outcomes generated from a set of nested rules. Further, prediction is a ubiquitous feature of nervous systems, a point emphasized in the general theories of predictive coding (Rao and Ballard, 1999) and the Bayesian brain (Friston, 2010). Thus, another important set of remaining questions concerns the unique characteristics of cerebellar predictive mechanisms. Current theoretical accountsbased on microcircuit anatomy, neuroimaging, and patient studies-suggest context specificity (Ramnani, 2006), automaticity (Ito, 2008), and precise timing (Ivry and Spencer, 2004) as potential constraints for characterizing the predictive capabilities of the cerebellum.

Some limitations with the present study need to be addressed. First, word frequency differed between conditions, with higher frequency words more likely to occur in the final position in the scrambled condition. However, we find it unlikely that the results can be attributed to word frequency effects since the main reported findings remained significant in the control analyses. Second, since we used fixed stimulus intervals, the BOLD response to the preceding sentence might have influenced the BOLD response to the target word. However, this cannot account for the difference between the incongruent and congruent sentences as these conditions only differed with respect to the final target word.

Third, the RT differences across conditions raise the possibility that the cerebellar activations were more related to differences in motor preparation than to differences in language processing. We addressed this concern by including trial-by-trial RTs and condition RT differences in the analyses, a procedure which proved sufficiently sensitive to detect effects of RT variability, including within some cerebellar foci. Nonetheless, the cerebellar activations related to both linguistic predictability as well as violations of linguistic predictions remained reliable even when we included the RT data as regressors in our model. Moreover, the cerebellar activations associated with word predictability were located in areas more closely associated with cognitive functions than motor control (Stoodley and Schmahmann, 2009).

Fourth, we recognize that word predictability is confounded, to some extent, with other variables; for example, predictable sentences are also likely to be experienced as more meaningful and incongruent target words are likely to not only generate an error signal, but also engage brain areas involved in sentence comprehension. Future studies will be required to examine the relationships between such variables as prediction, meaningfulness, error processing, and comprehension. We note, however, that our interpretation is consistent with results from numerous studies of cerebellar contributions to language, perception, and motor control. Thus, while alternative explanations cannot be conclusively ruled out, we believe that the generation of predictions in the cerebellum constitutes the most parsimonious account of the present findings.

Finally, and perhaps most challenging, the current results, along with those obtained in numerous other imaging studies demonstrating cerebellar activations during language tasks, stand in apparent contradiction to the clinical literature. Pronounced language comprehension deficits are not observed in patients with acquired cerebellar pathology (Alexander et al., 2012). However, it is important to note that the motor deficits observed in ataxia tend to become most evident during complex movements. By analogy, cerebellar predictions might aid, but in a strict sense not be essential for, language comprehension. As a case in point, while words can certainly be understood when presented alone, presenting them within a predictive context significantly aids comprehension; for instance, prediction can increase reading speed by allowing the reader to skip predictable words, or facilitate comprehension in noisy environments (Pickering and Garrod, 2007). Future neuropsychological studies should investigate the effects of cerebellar lesions on language comprehension in more complex contexts (such as noisy environments) or by comparing conditions in which the degree of predictability is manipulated.

In conclusion, we found that the predictability of visually presented words modulated the BOLD response in the posterior cerebellum. The observed activation patterns matched our expectations based on previous imaging studies of internal models in sensorimotor control. Thus our results are in line with the idea of a generalized role for the cerebellum in encoding internal models, a hypothesis that offers a unified perspective on cerebellar function in motor and non-motor tasks.

\section{References}

Albus JS, Branch DT (1971) A theory of cerebellar function. Math Biosci 10:25-61. CrossRef

Alexander MP, Gillingham S, Schweizer T, Stuss DT (2012) Cognitive impairments due to focal cerebellar injuries in adults. Cortex 48:980-990. CrossRef Medline

Allen G, Buxton RB, Wong EC, Courchesne E (1997) Attentional activation of the cerebellum independent of motor involvement. Science 275:1940 1943. CrossRef Medline

Ashburner J, Friston KJ (2005) Unified segmentation. Neuroimage 26:839851. CrossRef Medline

Balsters JH, Whelan CD, Robertson IH, Ramnani N (2013) Cerebellum and cognition: evidence for the encoding of higher order rules. Cereb Cortex 23:1433-1443. CrossRef Medline

Bastian AJ (2006) Learning to predict the future: the cerebellum adapts feedforward movement control. Curr Opin Neurobiol 16:645-649. CrossRef Medline

Bubic A, Cramon von DY, Jacobsen T, Schröger E, Schubotz RI (2009) Violation of expectation: neural correlates reflect bases of prediction. J Cogn Neurosci 21:155-168. CrossRef Medline

Buckner RL, Krienen FM, Castellanos A, Diaz JC, Yeo BT (2011) The organization of the human cerebellum estimated by intrinsic functional connectivity. J Neurophysiol 106:2322-2345. CrossRef Medline

Carreiras M, Mechelli A, Price CJ (2006) Effect of word and syllable frequency on activation during lexical decision and reading aloud. Hum Brain Mapp 27:963-972. CrossRef Medline

Chee MW, Hon NH, Caplan D, Lee HL, Goh J (2002) Frequency of concrete words modulates prefrontal activation during semantic judgments. Neuroimage 16:259-268. CrossRef Medline

Chein JM, Fiez JA (2001) Dissociation of verbal working memory system components using a delayed serial recall task. Cereb Cortex 11:10031014. CrossRef Medline

Chen SH, Desmond JE (2005) Cerebrocerebellar networks during articulatory rehearsal and verbal working memory tasks. Neuroimage 24:332338. CrossRef Medline

Chumbley JR, Friston KJ (2009) False discovery rate revisited: FDR and topological inference using Gaussian random fields. Neuroimage 44:6270. CrossRef Medline

Debruille JB, Renoult L (2009) Effects of semantic matching and of semantic category on reaction time and N400 that resist numerous repetitions. Neuropsychologia 47:506-517. CrossRef Medline

Desmond JE, Gabrieli JD, Glover GH (1998) Dissociation of frontal and cerebellar activity in a cognitive task: evidence for a distinction between selection and search. Neuroimage 7:368-376. CrossRef Medline 
Diedrichsen J (2006) A spatially unbiased atlas template of the human cerebellum. Neuroimage 33:127-138. CrossRef Medline

Diedrichsen J, Hashambhoy Y, Rane T, Shadmehr R (2005) Neural correlates of reach errors. J Neurosci 25:9919-9931. CrossRef Medline

Doya K (1999) What are the computations of the cerebellum, the basal ganglia and the cerebral cortex? Neural Netw 12:961-974. CrossRef Medline

E KH, Chen SH, Ho MH, Desmond JE (2014) A meta-analysis of cerebellar contributions to higher cognition from PET and fMRI studies. Hum Brain Mapp 35:593-615. CrossRef Medline

Friston K (2010) The free-energy principle: a unified brain theory? Nat Rev Neurosci 11:127-138. CrossRef Medline

Gagnepain P, Henson RN, Davis MH (2012) Temporal predictive codes for spoken words in auditory cortex. Curr Biol 22:615-621. CrossRef Medline

Glickstein M, Sultan F, Voogd J (2011) Functional localization in the cerebellum. Cortex 47:59-80. CrossRef Medline

Grande M, Meffert E, Huber W, Amunts K, Heim S (2011) Word frequency effects in the left IFG in dyslexic and normally reading children during picture naming and reading. Neuroimage 57:1212-1220. CrossRef Medline

Grinband J, Wager TD, Lindquist M, Ferrera VP, Hirsch J (2008) Detection of time-varying signals in event-related fMRI designs. Neuroimage 43: 509-520. CrossRef Medline

Grinband J, Savitskaya J, Wager TD, Teichert T, Ferrera VP, Hirsch J (2011) The dorsal medial frontal cortex is sensitive to time on task, not response conflict or error likelihood. Neuroimage 57:303-311. CrossRef Medline

Imamizu H, Kawato M (2012) Cerebellar internal models: implications for the dexterous use of tools. Cerebellum 11:325-335. CrossRef Medline

Imamizu H, Miyauchi S, Tamada T, Sasaki Y, Takino R, Pütz B, Yoshioka T, Kawato M (2000) Human cerebellar activity reflecting an acquired internal model of a new tool. Nature 403:192-195. CrossRef Medline

Ito M (2006) Cerebellar circuitry as a neuronal machine. Prog Neurobiol 78:272-303. CrossRef Medline

Ito M (2008) Control of mental activities by internal models in the cerebellum. Nat Rev Neurosci 9:304-313. CrossRef Medline

Ivry RB, Spencer RM (2004) The neural representation of time. Curr Opin Neurobiol 14:225-232. CrossRef Medline

Knolle F, Schröger E, Baess P, Kotz SA (2012) The cerebellum generates motor-to-auditory predictions: ERP lesion evidence. J Cogn Neurosci 24:698-706. CrossRef Medline

Knolle F, Schröger E, Kotz SA (2013) Cerebellar contribution to the prediction of self-initiated sounds. Cortex 49:2449-2461. CrossRef Medline

Kujala J, Pammer K, Cornelissen P, Roebroeck A, Formisano E, Salmelin R (2007) Phase coupling in a cerebro-cerebellar network at 8-13 Hz during reading. Cereb Cortex 17:1476-1485. Medline

Kutas M, Federmeier KD (2011) Thirty years and counting: finding meaning in the N400 component of the event-related brain potential (ERP). Annu Rev Psychol 62:621-647. CrossRef Medline

Lau EF, Phillips C, Poeppel D (2008) A cortical network for semantics: (de)constructing the N400. Nat Rev Neurosci 9:920-933. CrossRef Medline

Lesage E, Morgan BE, Olson AC, Meyer AS, Miall RC (2012) Cerebellar rTMS disrupts predictive language processing. Curr Biol 22:R794-R795. CrossRef Medline

Marr D (1969) A theory of cerebellar cortex. J Physiol 202:437-470. Medline

Meyer DE, Schvaneveldt RW (1971) Facilitation in recognizing pairs of words: evidence of a dependence between retrieval operations. J Exp Psychol 90:227-234. CrossRef Medline

Murdoch BE (2010) The cerebellum and language: historical perspective and review. Cortex 46:858-868. CrossRef Medline

Nicolson RI, Fawcett AJ, Dean P (2001) Developmental dyslexia: the cerebellar deficit hypothesis. Trends Neurosci 24:508-511. CrossRef Medline

Ortu D, Allan K, Donaldson DI (2013) Is the N400 effect a neurophysiological index of associative relationships? Neuropsychologia 51:1742-1748. CrossRef Medline

Pickering MJ, Garrod S (2007) Do people use language production to make predictions during comprehension? Trends Cogn Sci 11:105-110. CrossRef Medline

Pickering MJ, Garrod S (2013) An integrated theory of language production and comprehension. Behav Brain Sci 36:329-347. CrossRef Medline

Poeppel D, Emmorey K, Hickok G, Pylkkänen L (2012) Towards a new neurobiology of language. J Neurosci 32:14125-14131. CrossRef Medline

Price CJ (2010) The anatomy of language: a review of 100 fMRI studies published in 2009. Ann N Y Acad Sci 1191:62-88. CrossRef Medline

Ramnani N (2006) The primate cortico-cerebellar system: anatomy and function. Nat Rev Neurosci 7:511-522. CrossRef Medline

Rao RP, Ballard DH (1999) Predictive coding in the visual cortex: a functional interpretation of some extra-classical receptive-field effects. Nat Neurosci 2:79-87. CrossRef Medline

Ravizza SM, McCormick CA, Schlerf JE, Justus T, Ivry RB, Fiez JA (2006) Cerebellar damage produces selective deficits in verbal working memory. Brain 129:306-320. Medline

Rorden C, Karnath HO, Bonilha L (2007) Improving lesion-symptom mapping. J Cogn Neurosci 19:1081-1088. CrossRef Medline

Schlerf J, Ivry RB, Diedrichsen J (2012) Encoding of sensory prediction errors in the human cerebellum. J Neurosci 32:4913-4922. CrossRef Medline

Shattuck DW, Mirza M, Adisetiyo V, Hojatkashani C, Salamon G, Narr KL, Poldrack RA, Bilder RM, Toga AW (2008) Construction of a 3D probabilistic atlas of human cortical structures. Neuroimage 39:1064-1080. CrossRef Medline

Stoodley CJ, Schmahmann JD (2009) Functional topography in the human cerebellum: a meta-analysis of neuroimaging studies. Neuroimage 44: 489-501. CrossRef Medline

Strick PL, Dum RP, Fiez JA (2009) Cerebellum and nonmotor function. Annu Rev Neurosci 32:413-434. CrossRef Medline

Tseng YW, Diedrichsen J, Krakauer JW, Shadmehr R, Bastian AJ (2007) Sensory prediction errors drive cerebellum-dependent adaptation of reaching. J Neurophysiol 98:54-62. CrossRef Medline

Van Essen DC (2012) Cortical cartography and Caret software. Neuroimage 62:757-764. CrossRef Medline

Wolpert DM, Ghahramani Z, Jordan MI (1995) An internal model for sensorimotor integration. Science 269:1880-1882. CrossRef Medline

Wolpert DM, Diedrichsen J, Flanagan JR (2011) Principles of sensorimotor learning. Nat Rev Neurosci 12:739-751. CrossRef Medline

Xu J, Kemeny S, Park G, Frattali C, Braun A (2005) Language in context: emergent features of word, sentence, and narrative comprehension. Neuroimage 25:1002-1015. CrossRef Medline

Yarkoni T, Barch DM, Gray JR, Conturo TE, Braver TS (2009) BOLD correlates of trial-by-trial reaction time variability in gray and white matter: a multistudy fMRI analysis. PLoS ONE 4:e4257. CrossRef Medline 